\title{
Introducción. La compleja articulación de lo sustantivo y lo subjetivo en el ser humano. - De la antropología hegeliana a su antropología filosófica-
}

\author{
JUAN J. PADIAL \\ Universidad de Málaga
}

\section{TRAZANDo Rayuelas POR la ANTROPOlogía Filosófica de Hegel.}

El dolor y la alegría, el ansia y la satisfacción son pares de emociones opuestas, y pares en los que el ser vivo adquiere por primera vez cierto grado de interioridad subjetiva, un saber de su sustancialidad, antes opaca. En ellos no solo se encuentra cierta apertura, y claridad de su sí mismo sustancial, sino que su subjetividad se abre a otros. Quizá una de las primeras formas de esta última apertura en la escala de lo viviente sea la voz. La más alta, la palabra o quizá el canto. Todos estos afectos, y sus expresiones,

"superan el uno mismo individual; [el dolor y el ansia] son la conciencia de la contradicción, [la alegría y la satisfacción] el estado de vuelta en sî” ${ }^{1}$.

Vivir la contradicción o descansar en uno mismo no son meros eventos psicológicos privados. No son tan sólo la conmoción del viviente, sino su apertura. Por ello, el tratamiento hegeliano de los afectos anticipa $-\mathrm{y}$ supera en mucho, por cuanto no se restrige al valor para la supervivencia de los gestos expresivos - el que casi setenta años más tarde llevará a cabo Darwin, al tratar de La expresión de las emociones en el hombre y los animales. En cualquier caso, no hay dolor ni ansia, ni alegría ni satisfacción si no es como valoración de lo externo respecto del sí mismo, y por lo tanto, en la expresión de emociones y afectos se muestra claramente que el viviente no es un ser clausurado, sino abierto. Esta apertura implica que no hay subjetividad sin dominio sobre el tiempo y el espacio. Potestad sobre el espacio que se manifiesta en la mayor

1 Hegel, G.W.F., Filosofía real, Fondo de Cultura Económica, 1984, p. 140. 
o menor autonomía de movimientos del viviente. Dominio del tiempo que aparece en la voz de forma incipiente. Pero en la palabra, la exteriorización de la interioridad resulta plena. Un criterio para ordenar los grados de satisfacción proviene por tanto del grado de libertad frente a lo que hay. Y esta libertad es tanto mayor cuanto menor sea el encadenamiento a los trabajos y peligros. Por ello es que cabe hablar de una satisfacción de la contemplación, del sosiego, un levantar el vuelo a la caída de la noche, cuando todo ha pasado. Y ese alborozo, seguramente para Hegel, sería la más honda satisfacción y la más profunda alegría que cabe, aunque lejos del entusiasmo, y cercano al sosiego.

Pues bien una satisfacción semejante es la que me embarga al tratar de los trabajos reunidos en este primer volumen sobre la antropología filosófica de Hegel. El deleite de la contemplación, la fruición en el fruto de muchas voluntades mancomunadas en la ingente tarea de pensar filosóficamente al ser humano. Tarea desmedida, que no se acaba aquí, pero a la que se ha contribuido sin olvidar la réplica de Aristóteles al poeta Simónides, pues"es indigno del hombre no tensarse con todas sus fuerzas a lo que le es proporcionado"2.

Se recogen pues, en este libro una serie de contribuciones sobre la antropología filosófica de G. W. F. Hegel. Mas "serie" no arbitraria, mero encadenamiento o sucesión de textos. Se trata de una serie que ha devenido ordenada, pero en cuyo origen solo se puede situar la libertad; la libertad en la convocatoria que reunió a un nutrido grupo de muy buenos especialistas en el idealismo alemán. El vigor de la respuesta a la convocatoria fue ya un motivo para la dicha. Pero aún mayor contento proviene del constatar la profunda articulación que subyace a los textos, y la profundidad y nivel científicos de los mismos. Este libro no es algo así como una letanía de textos separados, aislados, y reunidos extrínsecamente mediante el fácil expediente de una encuadernación conjunta. Como podrá comprobar el lector, todas las contribuciones a este volumen se traban y relacionan entre sí. Es cierto que no de un modo elemental, pues aquí y allá hay voces críticas, que señalan una aporía o un problema. Pero no es menos cierto también, que aquí y allá se encuentran contribuciones que intentan resolver o enfrentarse precisamente con esas mismas aporías. Además concurren diversos acercamientos metodológicos. Los puntos de vista de la filosofía del espíritu u ontología hegeliana, los de la filosofía de la conciencia o fenomenología, y el acercamiento desde las objetividades culturales o filosofía del espíritu objetivo se entrecruzan en este volumen y el siguiente. Es por ello que la tarea del que introduce viene a ser la del trazado de una cartografía de las páginas que se están leyendo, o como gustaría decir a Julio Cortazar, la exposición de las diferentes rayuelas que se pueden trazar en estás más de ochocientas páginas. Y se trata de una labor necesaria por cuanto el orden entre los textos es extrínseco a los 
mismos, pues aunque reunidos por temas, se ha procedido a un amontonamiento alfabético entre los autores.

Todas las contribuciones a estos dos volúmenes tienen en común el ser aportaciones a la antropología filosófica, y por tanto, la temática es más amplia que el campo de temas que Hegel contemplaba como pertenecientes a la antropología sin más. En su Enciclopedia para los últimos cursos de Bachillerato, Hegel señala - con la extraordinaria claridad que le dio trabajar como redactor en un periódico local en Bamberg, y como director de un centro de enseñanza secundaria en Nüremberg - , que

"el espíritu considerado de por sí, hay que entenderlo en su existencia natural y en su conexión inmediata con el cuerpo orgánico y en su dependencia de las afecciones y estados del cuerpo orgánico, que de tal conexión inmediata dimana; es la antropología"3.

Aparece la antropología como el estudio del espíritu que comienza a desarrollarse desde sí mismo, un espíritu que despierta y se despabila desde condiciones que son naturales, como los estados de su cuerpo (despierto, dormido, sano, enfermo...) o su existencia natural (carácter, temperamento, personalidad cultural, etc.). Por lo tanto, lo que Hegel está considerando bajo la rúbrica "Antropología" es la comprensión filosófica de los aportes de ciencias como la medicina, la geografía o la etnografía. Así las cosas, la antropología filosófica no se corresponde exactamente con la sección de la filosofía titulada por Hegel como "antropología". Tampoco la filosofía del espíritu subjetivo correspondería a lo que hoy denominamos antropología filosófica, ni tan siquiera complementándola con la teoría del espíritu objetivo. Y no obstante, la antropología filosófica, - cuya carta de constitución está en la obra de Max Scheller y Ernst Cassirer - es plenamente consciente de la enorme deuda que tiene para con el pensamiento hegeliano. Sucede lo mismo con la contemporánea hermenéutica filosófica y en general con todas las ciencias del espíritu, tal y como hoy son cultivadas. Ya sea a través de Dilthey y Gadamer, ya a través de Max Weber, Niklas Luhman, o Émile Durkheim, la savia del pensamiento hegeliano ha nutrido - y sigue sustentando de hecho, aunque sea una sorpresa para muchos - las discusiones teóricas contemporáneas, como por otra parte muestran, a las claras, la obra de filósofos como Charles Taylor o John McDowell.

Urgía por tanto investigar estas fuentes de sentido, estos ocultos veneros que manan en muchas de las cuestiones más discutidas en nuestra actualidad. No hacerlo equivale a estar enterados de lo último, del x, y, z de nuestra disciplina,

3 Hegel, Enciclopedia filosófica para los últimos cursos del bachillerato, MuVIM, Valencia, 2007, § 129. 
pero ignorar el a, b, c, de la historia de nuestras ideas; se corre el riesgo, por tanto, de ignorar el planteamiento de nuestros problemas. Justo aquello que nos puede poner en condiciones de resolver adecuadamente las cuestiones que nos son más acuciantes. Es por ello, que la investigación sobre la antropología filosófica de Hegel resulta urgente, significativa, inexcusable e importante, porque equivale a elevar nuestras discusiones a su altura histórica, altura que pudiera ser hubiéramos perdido.

\section{LA ARTICULACIÓN DE LA SUSTANTIVIDAD Y LA SUBJETIVIDAD HUMANAS.}

Los temas de este volumen son la sustancialidad y subjetividad humanas, temas que hay que considerar no en su separación, sino en su conjunción. Lo importante es la "y", por ello Hegel habló de la dialéctica sustancia-sujeto. Como ha mostrado el profesor Juan García, lo distintivo de la consideración hegeliana de la subjetividad estriba en la necesidad de saber de sí propio de las realidades espirituales. No es lo definitivo en el análisis de Hegel la consideración de las facultades, los actos o los objetos. Su psicología filosófica está conservada, pero cancelada simultáneamente - aufhebt- en el tratamiento de lo que Dilthey caracterizaría como autognosis. De aquí que resulte tan paradójica la inclusión del capítulo sobre "fenomenología del espíritu" en la Enciclopedia de las Ciencias Filosóficas, como hace notar el profesor Polo en su texto. Y desde luego, lo que podríamos denominar psicología filosófica hegeliana no se atiene al plan trazado desde antiguo, desde la reformulación por Averroes y Avicena de la psicología aristotélica y la fundación de la psicología de las facultades. Para Hegel, entendimiento y voluntad, conciencia sensible y deseo se median entre sí, se vinculan dialécticamente, de modo que no cabe hablar estrictamente de facultades separadas, sino de los polos teórico y práctico de la subjetividad.

El tema de la subjetividad en Hegel hunde sus raíces en las reflexiones fichteanas sobre el yo y la posición de sí mismo, como concluye también el profesor Leonardo Polo en su artículo. De aquí la importancia de los temas de la mismidad, el ser para sí y la interioridad. "Lo que no es para sí mismo, no es Yo", sentenció Fichte ${ }^{4}$. Se publica en este volumen un texto del prof. Reinhard Lauth $(† 2007)$, para mí de imborrable recuerdo, quien en su calidad de presidente de la Fichte-Kommission en la Academia Bávara de las Ciencias, quizá fuese una de las voces más autorizadas para hablar de la relación de Hegel con Fichte. Su diagnóstico no puede ser más crítico con la posición hegeliana. No queremos, con este volumen, dejar de filosofar o mostrar de un modo acrítico las bondades de la antropología filosófica de Hegel. Por ello, lejos de las filiaciones de escuela filosófica, o de los monografías meramente expositivas, este

4 Fichte, GA I/2, 260. 
libro es una incitación al discernimiento filosófico.

El excelente estudio del profesor Jacinto Choza de la Universidad de Sevilla, tiene entre sus muchas virtudes, la de abordar la articulación de los planos metodológicos con los que se podría reconstruir la antropología filosófica de Hegel. El plano de la interioridad subjetiva se desdobla en un plano en el que se trata de la libertad subjetiva, la individualidad y la conciencia y otro en el que se tematiza el espíritu en su dimensión más propia, que para Hegel es el concepto.

Este segundo plano, propiamente especulativo o metafísico, forma parte inexcusable de la investigación hegeliana sobre la subjetividad y el tiempo, como ha mostrado el profesor Ignacio Falgueras en su contribución. Me atrevo a subrayar no sólo la hondura de la investigación llevada a cabo por Ignacio Falgueras, sino también la belleza que tiene la cadencia de su razonar. Trata su estudio de exponer por qué el yo y el tiempo son conceptos según Hegel, el uno existente, el otro en su exterioridad. Al hacerlo se advierte por qué la lógica posee un alcance especulativo, y se está en condiciones de advertir las repercusiones antropológicas de la especulación moderna. Centrarse en el carácter especulativo del sistema tiene el interés de mostrar la unidad de la Ciencia de la lógica, pues Hegel intensifica la reflexión moderna. No se arredra ante la escisión, sino que intenta conciliar los opuestos. A esto responde la distinción entre las dos negaciones hegelianas. La lógica de la esencia y la lógica del concepto, se comprenden así en su estricta continuidad, y por lo tanto también se comprende la relevancia del concepto, del lugar especulativo-metafísico de la conciliación, el universal-concreto, en el que cualquiera de los opuestos aparecen, el uno al mirar el otro y viceversa. Pues bien, este poder de la negación es de suma importancia en la antropología filosófica y la filosofía de la historia hegelianas, como muestra el profesor Falgueras en su dilucidación del sentido hegeliano de la alienación de la Idea absoluta.

El tema de la alienación de la Idea, y la unidad del corpus hegeliano ha sido también el tema sobre el que versa el trabajo del profesor Juan A. García, también de la universidad de Málaga. Consideraciones más amplias sobre la unidad en Hegel las proporciona el texto inédito del profesor Leonardo Polo que se publica en este volumen. En cualquier caso, el enfoque de estos autores es el de la antropología metafísica. Orientación de suma relevancia para Hegel, y me atrevería a afirmar que para la antropología filosófica en general.

Que para Hegel, la clave de arco de su pensamiento se encuentra en la Ciencia de la Lógica, y en concreto en su tercer volumen, La doctrina del concepto, es algo que se encarga de señalar, también desde el punto de vista ontológico,el profesor Mariano Álvarez-Gómez de la Universidad de Salamanca. Su magistral exposición apunta al momento decisivo del filosofar hegeliano, el instante de su ¡Eureka!, el momento de resolución de las más intensas aporías de su pensar. 
Es cierto que la trayectoria intelectual de Hegel está jalonada por diversos hitos o puntos de inflexión. Así el encuentro con Hölderlin en Frankfurt supuso la búsqueda de la totalidad, y un quiebro en las investigaciones político-críticoreligiosas acometidas hasta entonces. La colaboración con Schelling en Jena le permitió investigar bien el edificio crítico y el fichteano, pero al precio de trabajar con el esquema de la doctrina schellinguiana de las potencias. Y ese fue un precio muy caro, como se nota en la Fenomenología. Pero el momento en que Hegel habla con voz propia viene a ser, justamente la Ciencia de la lógica, y en concreto los pasajes analizados por el profesor Álvarez-Gómez. Allí se resuelven los muchos años de heurística sobre el amor, la vida, el poder, y la configuración sistémica del idealismo. Allí aparece el sentido hegeliano de lo universal y de la Idea.

\section{SUEÑo (DEL ESPÍRITU).}

Si en lugar de adoptar la perspectiva especulativa o absoluta, la del significado de la Idea y el de su alienación, nos situamos en una perspectiva que permita asistir a la aparición de la subjetividad finita, del yo, entonces será necesario indagar qué significa tal aparición, y su relación con el mundo, con la naturaleza. Este es el cometido del excelente trabajo del profesor Edgar Maraguat, de la Universidad de Valencia. Al hacerlo se prosigue con los hallazgos sobre la unidad de la antropología filosófica de Hegel, pero ahora sentando la articulación de la parte lógica del sistema, con las dos partes reales, o sea, la filosofía de la naturaleza y la del espíritu, y los vínculos entre estas dos ciencias filosóficas reales. Dicho con otras palabras, se intenta responder qué significa para la naturaleza ser la presuposición del espíritu, y para el espíritu, ser la verdad de la naturaleza. Al hacerlo se aclaran decisivas cuestiones de la sustancialidad humana.

Esta es la clave de la antropología para Hegel. Más que ciencia del espíritu subjetivo, es ciencia de su despertar, de su aparecer, de las primeras articulaciones entre naturaleza y espíritu. Si para Leibniz las mónadas se distinguían según sus grados de claridad, tales grados, son los niveles en que la sustancialidad va siendo acogida, o los grados en que el sujeto se posee a sí mismo. Por tanto tales grados de claridad son inseparables del proceso dialéctico, o de la experiencia de la conciencia. Este asunto ha sido el objeto de estudio de Federico Sanguinetti, de la Universidad de Pisa en Italia.

El profesor José Ignacio Murillo, de la Universidad de Navarra y el profesor Andrés Alonso Martos de la Universidad de Valencia también insisten en los temas de la sustancialidad, el primero al tratar de lo psíquico - das Psychischen Hegel y Scheller, el segundo al tratar de lo que podría ser un acercamiento al Gesammtorganismus. En uno y otro estudio aparece la inquietud hegeliana por estudiar el conjunto, el organismo físico-psíquico-mental, o con otras palabras 
la promoción integral de lo humano, no tan sólo de los ápices de lo humano, tal y como aparece en la obra de Kant, en el que la distancia que se abre entre lo natural - los deseos - y lo espiritual - la razón - es insalvable. Respecto de un tratamiento de los ápices humanos como el kantiano, que lleve a afirmar que lo único santo dentro y fuera del mundo sea una voluntad buena, Hegel se pronunciará señalando la finitud de tal voluntad; buena tan solo en su independencia, en su soledad, en su distancia respecto a todo lo demás humano, como los deseos y motivos, que quedan así condenados por Kant. Por ello, la investigación sobre la afectividad (Murillo) o sobre el magnetismo animal (Alonso Martos), tienen como finalidad la advertencia del modo en que la naturaleza es subsumida por el espíritu; no siendo tal subsunción una lucha contra ella, extremo en el que se resuelve la moral kantiana.

En mi propia contribución a este volumen, he estudiado la religiosidad del joven Hegel como clave de su antropología filosófica. Religiosidad que viene a ser una respuesta a la Ilustración alemana y a la metafísica postulativa kantiana. Estos dos momentos de la historia del pensamiento comparten el fracturar internamente al hombre, y el separarlo del resto de la realidad. Aquí el punto de vista adoptado está a caballo entre la consideración ontológica y la de la filosofía de la conciencia. Entre el punto de vista de la interioridad objetiva y la subjetiva, y por lo tanto permite transitar gradualmente a ésta última perspectiva.

El profesor Daniel Brauer ha llamado insistentemente la atención sobre el carácter de permanente ajuste que realiza la conciencia humana entre el mundo, la interioridad humana y ella misma, o dicho de acuerdo con la metáfora que Hegel gustaba utilizar, que el sacrificio de sí es necesario para que la subjetividad se posesione de sí. De aquí que Hegel pudiera afirmar en la Filosofía real jenense que "la enajenación sea mi necesidad" , y en otro orden de cosas, que la locura sea un privilegio de la autoconciencia, como ha explicado el prof. Brauer. También Gillaume Lejeune ha explorado magistralmente el tema de la alienación mental y de su tratamiento. La investigación de Lejeune se centra en una rectificación de la Historia de la locura de Foucault, mostrando que para Hegel son factores que propician la locura tanto los subjetivos como los objetivos, esto es, los culturales. Es así como se conecta el yo con el tiempo en esta temática, la intemporalidad del espíritu con la temporalidad de la Sittlichkeit.

Como Brauer sostiene, la índole de la autoconciencia posibilita la demencia, como un trastorno del permanente contraste entre el sí mismo y el mundo. Un quedar fijado del yo, aislado, separado. Es por ello que Hegel hable del sacrificio del sí mismo, de la importancia del reconocimiento o de la enajenación, en un sentido tanto patológico como no patológico. Temas estos, que aunque equi- 
valentes, pueden ser tratados desde diversos puntos de vista. Brauer y Lejeune inquieren por las anomalías - patologías - de la subsunción de lo natural o cultural en el espíritu. Esta subsunción de lo natural es realizada tanto en la dimensión sustancial como en la subjetiva del espíritu finito. Sustancialmente la naturaleza es subsumida por el espíritu en forma de hábitos; subjetivamente en el lenguaje. Hábito y lenguaje en Hegel constituyen la parte central del estudio del profesor Jacinto Choza.

\section{INTERIORIZÁNDOSE EN LA PROPIA EXTERIORIDAD: EL ESPÍRITU TEÓRICO.}

La profesora María del Carmen Paredes-Martín (Universidad de Salamanca) atiende también a la dimensión de la finitud en la antropología filosófica de Hegel. Su incisivo y perspicaz estudio sobre la relación entre vida humana y entendimiento puede muy bien servir de pórtico a la sección sobre la experiencia y la conciencia de este libro; sección ésta, la que más directamente tiene que ver con el conocimiento humano en cuanto que inserto en la vida de los seres dotados de autoconciencia. No se trata por tanto de una sección sobre teoría del conocimiento, sino sobre antropología filosófica, donde lo crucial es la experiencia que la conciencia realiza en su transcurso temporal. Vuelve de nuevo a aparecer el tema-marco de la relación entre el yo y el tiempo.

El tomar posesión de algunos aspectos de la propia sustancialidad que se logra mediante los hábitos, o la vivencia de la distancia respecto de sí mismo, la vivencia de una escisión, implican que la subjetividad humana no puede ser considerada como cosa, res o sustancia. Tal tratamiento resulta común a la antigua psicología de las facultades, o la moderna psicología racional de Leibniz y Wolf, que penetra en el descubrimiento cartesiano de la res cogitans. Pero tratar de la conciencia, la razón o la voluntad en términos de eventos, procesos, etc., implica cometer un error categorial; o en términos hegelianos, equivale a realizar un juicio infinito, esto es una comparación entre términos incomparables. Error categorial por cuanto supone tal psicologismo que hay procesos psíquicos por lo mismo que constan los físicos. Esta observación hegeliana, de profunda raigambre aristotélica, le llevará a negar la distinción de facultades, y es la clave de la teoría del conocimiento hegeliana. La pluralidad de facultades le parece a Hegel una fragmentación de la subjetividad. Más que de diversas potencias, cada una conmensurada con objetos propios a través de operaciones peculiares, Hegel entiende que la actividad intelectual y volitiva se ahonda progresivamente, o que hay grados de claridad, que no definen sustancias diversas, como había pensado Leibniz, sino niveles en los que el espíritu toma posesión de sí, de interiorización, o de asunción de lo externo. Así el grado mínimo anticipa el máximo, y logrado éste, se puede tirar la escalera por la que se había subido, pues aquellas anticipaciones quedan depuradas y cumplidas en lo que ahora se ha logrado. Es por ello que tal ascensión, o tal conquista de claridad, 
viene a ser una experiencia para la conciencia, y por lo tanto, el conocimiento no es lo separado, sino aquello que realiza la experiencia en la que el espíritu se conquista. La tercera sección de este libro trata estos temas. El tirar de la escalera o la relación de la razón para con la imaginación, según Alejandro Rojas, la unidad que subyace a la distinción de actividades mentales, ha sido estudiada por Jaime Galván y Daniel Pérez. Óscar Cubo y Giuseppe Tufano tratan de lo ganado al tirar la escalera. Si sustituimos la metáfora wittgensteniana por la hegeliana, entonces habría que decir que el resultado es conclusión - silogismo, Zusammenschliessen - , aquello que muestra la unidad de los juicios - las divisiones originarias, Urteilen-.

Sobre la conclusión que la razón significa para lo anticipado en la imaginación trata el trabajo de Alejandro Rojas, de la Universidad de Málaga y actualmente becario Humboldt en la Universidad Ludwig Maximiliam de Munich. Estudia la imbricación de facultades como el deseo, la imaginación, o el entendimiento en el proceso racional, y la relación entre saber de sí e imaginación en la experiencia que realiza la autoconciencia. Sobre estas relaciones también ha realizado interesantísimas aportaciones Žižek, como señala en su texto Luis Felip López-Espinosa, y que son especialmente relevantes para entender el rol coprincipial de lo sustantivo respecto de lo subjetivo en el ser humano.

El proceso de crecimiento de la autoconciencia, el momento en que de una figura se transita a otra, la experiencia que la conciencia hace del mundo, ha sido analizado por Jaime Galván, de la State of New York University. Pero esta experiencia es siempre un interacción, en que lo nuevo conocido refluye sobre el horizonte de experiencias posibles que denominamos mundo, y lo transforma. Nunca es una experiencia unilateral, como si fuera únicamente un aprendizaje, algo que afecte a la conciencia, sin más, sino una interacción. Tal interacción ha sido analizada en detalle por Daniel Pérez a lo largo de algunas figuras de la Fenomenología del Espíritu.

Óscar del Cubo, de la UNED (España) ha tenido el acierto de enfocar la experiencia de la autoconciencia, no in actu exercitu, sino al modo de la ciencia, si seguimos la distinción aristotélica entre el acto según la ciencia y según su ejercicio. La ciencia para Hegel se gana al final de la Fenomenología, y permite conocer lo verdadero y lo falso de cada una de las figuras por las que la autoconciencia ha transitado. Al hacerlo se adquiere el conocimiento de los límites de la conciencia, y la necesidad de aquellas formas de autoconciencia cierta, que en modo alguno dudaba de su contenido, aunque lo que se presentaba estaba marcado por la falsedad. Lo falso había de ser admitido necesariamente en el proceso, pero la autoconciencia no lo sabía. El logro de la ciencia, como pone de relieve el profesor del Cubo es la anulación de la diferencia entre lo ya sabido y la verdad.

Una interesante propuesta para la conexión entre la teoría del conocimiento 
hegeliana y las contemporáneas teorías de la conciencia, como las de Dennett, Chalmers o Nagel, puede encontrarse en la formulación matemática de algunos temas hegelianos realizada en este volumen por Edouard Asseo.

Del rédito para la antropología filosófica del saber absoluto también trata Giuseppe Tufano, de la Universidad de Salamanca. Realiza, siguiendo a Kojève, una lectura en clave antropológica, que no metafísica, de la Fenomenología del espíritu. Es así como este autor acierta a conectar filosóficamente algunos temas esbozados en el periodo bernés de Hegel con los desarrollados en Jena. En concreto, la manifestación del espíritu en la sensibilidad, en la que ya se anuncia, pero como mera anticipación, que se desvela y cobra su verdad en las figuras del sabio - el primer ideal, ilustrado y romántico, de educador moraly el saber absoluto.

\section{LA ACCIÓN Y EL ESPÍRITU PRÁCTICO.}

La necesidad con la que lo falso ha de ser admitido tiene su correlato en las formas de vida, como ha sido visto por el prof. Julio de Zan, de la Universidad Nacional de San Martín (Argentina). Cabe afirmar que el desgarramiento, la separación y el distanciamiento son necesarios para la conquista de la autonomía por parte del sujeto, pues sin ellos es imposible el tránsito de la familia a las formas políticas de organización social. Pero sin el reconocimiento la escisión quedaría irresoluta y la interioridad rota. Esta necesidad es más honda de lo que en principio pareciera, pues caracteriza por completo a la finitud. Como indica en su excelente texto el prof. Robert Williams de la Universidad de Illinois en Chicago, la libertad humana y el reconocimiento son por una parte, indisolubles, pues la conciencia es tanto relación consigo como relación con otros. Por otra parte la libertad y la existencia humanas tienen siempre una dimensión trágica, inseparable de la finitud, de la necesaria experiencia que rige la conciencia humana, y de las mediaciones histórico-culturales en que nacen situados siempre los seres humanos. Al examen de la relación, consigo y con los otros, a la dependencia del otro para la constitución de la relación consigo mismo, y a la inserción de la libertad en tal proceso, dedica el prof. Williams su contribución.

La importancia del periodo hegeliano de Jena para la antropología filosófica ha sido subrayada por muchos autores, como Honneth o Ricoeur. Williams, y Julio de Zan ahondan en el significado antropológico del reconocimiento, llevando la investigación más allá del interés ético que mueve a Ricoeur, al tratamiento del reconocimiento como de un a priori de la génesis y constitución de la subjetividad. Así para Williams, la intersubjetividad no es cumplidamente conflictual, sino más bien la realización del estar cabe sí en el otro, realización que es trágica de suyo, pero que mide la potencial grandeza humana. El análisis del reconocimiento elaborado por Sebastian Ostritsch (Universidad de Stuttgart) 
ofrece una alternativa hegeliana en el debate determinismo-compatibilismo.

A los presupuestos ontológicos del reconocimiento - la tematización de la sustancia espiritual como anhelo, fuerza e ímpetu - dedica el profesor José Manuel Sánchez, de la Universidad de Castilla-La Mancha, su texto, que permite vislumbrar la raíz de la negativa hegeliana a separar la inteligencia de la voluntad. La identificación leibniziana de la mónada con el entender y el apetecer, aparece en Hegel de nuevo, pero de modo tal que el sujeto pierde cualquier valor de fundamento. La sustancia no precontiene, como la mónada leibniziana, en su plenitud eterna la totalidad de sus predicados, sino que en lo otro, en su aparecer como lo opuesto de sí, en la escisión, cabe reconocer ulteriormente que se encontraba en sí mismo. La razón ya no es causa, y su papel ha de ser ahora asumido por la libertad y el reconocimiento, como se verá en la siguiente sección.

Entre el realismo de Jacobi y el idealismo trascendental se desenvuelve la posición antropológica de Hegel, como ha puesto de relieve Manuel Barrios en su contribución. Analiza preferentemente los escritos hegelianos anteriores a la Fenomenología, como sucesivas profundizaciones en el modo de suturar las escisiones que la modernidad produce por doquier. Tal gigantesco esfuerzo por la conciliación de un hombre desgarrado está al servicio de una idea de sujeto que no coincide sin más con el sujeto moderno, como señala magistralmente el profesor Barrios. Hegel ha disuelto o nihilizado tal forma de subjetividad cuyo correlato son las éticas universalistas del deber; o mejor aún, ha encarnado e insertado al yo en sus tradiciones. Vuelve a aparecer el tema-marco de estos dos volúmenes, el yo y el tiempo. Con suma perspicacia el doctor Barrios hace ver que la crítica de Hegel no va dirigida meramente contra el alma bella o la subjetividad moderna, sino que se puede dirigir contra las formas de identidad postmodernas; posiciones que presuntamente parecen haber abandonado las coordenadas vitales y reflexivas de la modernidad. Carlos Ortiz de Landázuri también insiste en la importancia de la perspectiva hegeliana para el enjuiciamiento de algunas posturas postmodernas.

Quizá la escisión más dolorosa para Hegel fuese la que atañe a la teoría kantiana de la acción, aquella contradicción entre la razón práctica y los motivos, entre lo personal y los impulsos. El texto de Christopher Yeomans de la Universidad de Purdue en los Estados Unidos, incide sobre el carácter indeterminado y maleable que tienen los motivos o impulsos para Hegel. Tal indeterminación es consecuencia directa de la negativa hegeliana a separar las facultades. Voluntad y tendencias no se relacionan como las piezas separadas de un circuito, sino que la dinámica tendencial humana muestra su plasticidad al permitir que las tendencias y deseos naturales sean asumidos y dirigidos desde la subjetividad. Lo humano consiste en que incluso lo biológico, y con ello toda la sustancialidad, no es autónomo, sino que está articulado e incluso 
coprincipiado desde la subjetividad. Esta asunción era el núcleo de los estudios tratados en la sección de este libro sobre la sustancialidad humana, y con los que el artículo del profesor Yeomans guarda estrecha afinidad. Si el profesor Choza había mostrado el papel del hábito en la articulación entre libertad y naturaleza, Yeomans concreta una de sus formas, aquella en la que la libertad informa las tendencias y motivaciones.

Dylan Lott, también de la Universidad de Illinois en Chicago, también ha centrado sus esfuerzos, en la dialéctica entre subjetividad y sustancialidad. Si el profesor Choza dilucidó el papel de los hábitos, una de las piezas clave de la sección "Antropología" de la Enciclopedia, Lott estudia el papel de la sustancialidad asumida en la subjetividad. Su texto tiene un enorme interés para las teorías psicológicas de la personalidad, en concreto con las corrientes personalistas sobre la génesis y constitución de la personalidad, en las que la memoria, lo vivido, las formas de vida, la educación recibida o la historia en la que uno es recibido y que continúa, conforman el coro de voces que constituyen la personalidad individual, al modo de lo que Husserl podría llamar una síntesis pasiva, sustancial, casi diríase que inconsciente. En la misma dirección apuntan los textos sobre la dignidad de la persona escritos por Javier Aguirre Román, de la Universidad Industrial de Santander en Colombia, y de Luis Gálvez, de Granada, España.

Entre las virtudes de Hegel siempre habrá que recordar su profunda afabilidad y la alegría con que gustaba de las reuniones sociales y de amigos. Y entre sus aficiones, una que cultivaría toda su vida, sería la de los juegos de naipes. No dejó de reflexionar sobre ello, quizás porque la "gente de profundo talento y brillante imaginación sea con frecuencia mal jugadora"6 . Desconozco si este reproche podía dirigirse a él mismo, pero aunque lo dudo, no dejaría de confesar que me gustaría que así hubiera sido, como mostrando en su propia vida la función del universal, que concilia afición y disposición. En cualquier caso, en estos pasatiempos Hegel veía que el "entendimiento y la pasión son las propiedades del alma que intervienen ahî" "Tal articulación entre factores subjetivos y sustantivos, entre el yo, y lo que el tiempo ha hecho de él, es lo que se investiga en las muchas páginas de este primer volumen sobre la antropología filosófica de Hegel. Ojalá contribuyan no solo a nuestro saber, sino también a nuestra mejoría en humanidad, aunque no tengamos conciencia ninguna de tal perfeccionamiento, y esté quede siempre sepultado pero operativo en la noche de nuestra sustancialidad.

6 Hegel, G. W. F., "Jugar a las cartas" en Escritos de Juventud, Fondo de Cultura Económica, México, 1978-1998, p. 253.

7 Ibidem. 